\title{
Producción y evaluación de tareas matemáticas en las herramientas CalcMe y GeoGebra con los futuros docentes de Educación Primaria
}

| Production and evaluation of mathematical tasks in the tools CalcMe and GeoGebra with future teachers of Primary Education |

\author{
Mónica Arnal-Palacián \\ marnalp@unizar.es \\ Universidad de Zaragoza \\ Zaragoza, España
}

Resumen: Este documento describe la experiencia realizada con las aplicaciones educativas y tecnológicas CalcMe y GeoGebra con futuros profesores asistentes al Grado de Educación Primaria, en las asignaturas Matemáticas y su Didáctica II y Matemáticas y su Didáctica III, durante los cursos lectivos 2018-2019 y 2019-2020. La demanda de este alumnado acerca de las soluciones de los ejercicios matemáticos que se les plantean y la necesidad de crear tantos ejercicios como requieran, propicia el uso de nuevas herramientas, en el trabajo autónomo del alumno tanto en el aula como fuera de ella. Cada alumno, tras la formación recibida en el aula, fue capaz de autocorregirse las tareas propuestas por la docente, así como elaborar tantos ejercicios nuevos como necesitase y adaptarlos a su propio aprendizaje. Todo ello potenció las habilidades didácticas del futuro docente.

Palabras Clave: GeoGebra, CalcMe, Didáctica de las Matemáticas, Aplicaciones educativas y tecnológicas.

\begin{abstract}
This document describes the experience carried out with the educational and technological applications CalcMe and GeoGebra, with future teachers attending the Degree in Primary Education, in the subjects Mathematics and its Didactics II and Mathematics and its Didactics III, during the academic years 2018-2019 and 2019-2020. The demand of these students about the solutions of the mathematical exercises that are presented to them and the need to create as many exercises as they need encourages the use of new tools, in the student's autonomous work both in and out of the classroom. Each student, after the training received in the classroom, was able to self-correct the tasks proposed by the teacher, as well as develop as many new exercises as needed and adapt them to their own learning. All this enhanced the teaching skills of the future teachers.
\end{abstract}

Keywords: GeoGebra, CalcMe, Didactics of Mathematics, Educational and technological applications.

\section{Introducción}

Debido al alto número de matriculados que asisten a las distintas asignaturas de un grado universitario, imposibilitando una individualización del aprendizaje de manera tradicional, y la disparidad 
de conocimientos matemáticos previos a la realización de la materia, surge la necesidad de adaptar el proceso de enseñanza-aprendizaje a cada uno de ellos, en aspectos aritméticos, geométricos y analíticos.

Además, pese a que los estudiantes universitarios del Grado de Educación Primaria están habituados, por motivos generacionales, a las nuevas tecnologías, desconocen en multitud de ocasiones las oportunidades que pueden desarrollarse con ellas en el ámbito de la didáctica en general y de Didáctica de las Matemáticas en particular. Estas aplicaciones, en la mayor parte de los momentos, están pensadas para su futuro alumnado, obviando en algunos instantes que también pueden serles de utilidad durante su etapa universitaria para la adquisición de conocimiento.

Durante todo el cuatrimestre en el que se desarrollan las asignaturas de Matemáticas y su Didáctica II y Matemáticas y su Didáctica III del Grado universitario en Educación Primaria, el alumnado demanda las soluciones y resoluciones de los ejercicios matemáticos que se plantean en el aula, además de surgir la necesidad de generar tantos ejercicios como fuesen imprescindibles en su propio proceso de aprendizaje, propiciando el uso de nuevas herramientas, tanto en el aula como en el trabajo autónomo del alumno fuera de ella. Por todo ello, en este documento se describe una experiencia en las que se utilizan las aplicaciones tecnológicas CalcMe y GeoGebra para abordar nociones matemáticas de aritmética y geometría.

Por todo lo anterior, se llevó a cabo la propuesta proponiendo los siguientes objetivos:

- Adquirir el conocimiento matemático de las asignaturas Matemáticas y su Didáctica II y III del Grado de Educación Primaria.

- Mejorar la actitud de los alumnos ante la incertidumbre de un resultado correcto en una tarea matemática.

- Facilitar la adquisición de algunas nociones matemáticas adaptando el aprendizaje a cada alumno.

- Desarrollar competencias digitales en los futuros docentes de Educación Primaria.

\section{Marco teórico}

\subsection{Antecedentes}

Los procesos formativos deben transformarse hacia las nuevas realidades de la sociedad de conocimiento actual. En esta transformación se encuentra la introducción de las Tecnologías de la información y la comunicación (TIC), influyendo en dos aspectos principales: por un lado, la relación y la interacción entre las personas en un intercambio de información y experiencias y, por otro lado, el acceso a una gran cantidad de información, buscando mejorar la calidad educativa (Flórez et al., 2017).

Lamentablemente, el acceso del profesorado y de su alumnado a las TIC está todavía lejos de cumplirse en muchos países, escuelas y aulas, por lo que todavía deben continuar el esfuerzo en esta dirección (Suasnabas et al., 2017).

En España, la utilización y manejo de las TIC forman parte del día a día de la vida del alumnado del Grado de Educación Primaria, aunque en muchos casos todavía no esté tan presente en el aula universitaria como fuera de ella (Tirado \& Roque, 2019).

Las TIC favorecen la adquisición de algunas nociones, incluso llegando a mejorar el rendimiento del alumnado de cualquier nivel educativo. Incluso, hay autores como Huertas y Pantoja (2016) que aceptan que se aprende mejor usando el ordenador que en un aula normal, permitiendo, además, que el

Producción y evaluación de tareas matemáticas en las herramientas CalcMe y GeoGebra con los futuros docentes de Educación Primaria. Arnal-Palacián, M.

Derechos Reservados @ 2022 Revista digital Matemática, Educación e Internet (https://revistas.tec.ac.cr/index.php/matematica) 
proceso de aprendizaje de manera sea individual (Saputra \& Fahrizal, 2019) e interpretar y experimentar por simulación (Ngnoulaye \& Lepage, 2018). Asimismo, a nivel institucional, la UNESCO (2014) recomendó promover las TIC en los centros educativos para favorecer la competencia tecnológica involucrada en los procesos de enseñanza-aprendizaje.

Fernández y Muñoz (2007) afirman que los alumnos que muestran un comportamiento disruptivo en el aula tienden a mostrar una mejor actitud cuando se hace uso de herramientas tecnológicas para el aprendizaje. Facilita la atención a la diversidad, creando actividades adaptadas a cada alumno y permitiendo que cada uno lleve su propio ritmo de trabajo. Sin embargo, Pascual (1998) advierte que el uso del ordenador no supone una mejora si no va acompañado de un adecuado planteamiento metodológico.

Con base a lo expuesto, en relación al uso de la TIC, se realizó una propuesta en la que se utilizaron los softwares matemáticos GeoGebra y CalcMe.

\subsection{GeoGebra}

GeoGebra fue creado en 2001 en el departamento de Didáctica de las Matemáticas de la Universidad de Salzburgo por Markus Hohenwarter, con la función de realizar construcciones geométricas y analíticas de forma sencilla.

Este software educativo combina las representaciones gráficas y las simbólicas al mismo tiempo, permitiendo la introducción de números, puntos, ecuaciones, funciones, etc. (Losada, 2008).

Arranz et al. (2011) lo destacan sobre otros programas debido a su facilidad, conexión y posibilidad de realización de construcciones dinámicas e interactivas; mientras que García-Cid et al. (2020) afirman que los estudiantes para maestro fomentan su autonomía y destrezas muy importantes en su futuro como docentes.

La gratuidad de GeoGebra, tanto para centros educativos, como para los alumnos, lo hace accesible a cualquier individuo. Estas ventajas se reflejan en la multitud de recursos que podemos encontrar en Internet. En un alto porcentaje, estos recursos están focalizados en la geometría, tanto en Educación Primaria como en Educación Secundaria, aunque en los últimos años han surgido investigaciones en el análisis matemático, como son los estudios de Hernández (2013), Tamayo (2013), Castillo et al. (2013) y Arnal et al. (2018).

Para que GeoGebra pueda introducirse y utilizarse de forma efectiva con fines docentes, debe integrar geometría, álgebra y cálculo de manera simultánea. En su uso se obtendrán mejores o peores resultados según la utilidad que se dé del mismo (Benites et al., 2009).

Iranzo y Fortuny (2009) utilizaron GeoGebra en su estudio por tratarse de un software intuitivo que no requiere estrategias de uso avanzadas para utilizarlo en el contexto de la investigación. En sus estudios llegaron a la conclusión de que GeoGebra les ayudaba a visualizar el problema y a evitar los obstáculos algebraicos. Además, el programa facilitaba un soporte visual algebraico y conceptual para la mayoría de los alumnos.

\subsection{CalcMe}

CalcMe es una aplicación en línea que ofrece un sistema de cálculo y representación gráfica. Está basada en Javascript e integrada en Wiris. A su vez, Wiris es un programa de álgebra computacional con propósitos educativos.

El uso de Wiris en general y CalcMe en particular en las aulas es mucho menos numeroso que en el

Producción y evaluación de tareas matemáticas en las herramientas CalcMe y GeoGebra con los futuros docentes de Educación Primaria. Arnal-Palacián, M.

Derechos Reservados @ 2022 Revista digital Matemática, Educación e Internet (https://revistas.tec.ac.cr/index.php/matematica) 
caso de GeoGebra, a pesar de ser también gratuito. Esto se refleja también en el ámbito de la investigación, donde son escasas las experiencias llevadas a cabo. Entre ellas se encuentran las realizadas por Acosta (2017).

El referenciado autor afirma que los docentes universitarios deben aplicar el software Wiris para mejorar el aprendizaje de sus estudiantes, mostrando facilidad en el manejo de esta herramienta. Para ello debe ofrecerse formación permanente a los docentes de matemáticas en su uso y aplicación, así como en diversos softwares. Además, recomienda el uso de esta aplicación en los estudiantes de nivel universitario para sus tareas de resolución de problemas, principalmente en la transformación de la información; realizando cálculos, análisis, síntesis, creatividad de modo fácil, motivado, dinámico, interactivo y también con ayuda de plataformas virtuales.

La experiencia de Ruiz et al. (2007) fue un esfuerzo por introducir modelo algebraico en la escuela secundaria y destacar el papel que puede desempeñar una calculadora simbólica como es el caso CalcMe.

\section{Metodología}

A continuación, se encuentran la descripción de la muestra, formada por estudiantes que cursan las asignaturas de Matemáticas y su Didáctica del Grado de Educación Primaria, las percepciones de este alumnado y el desarrollo de la propuesta efectuada.

\subsection{Muestra}

La muestra estuvo compuesta por los estudiantes del Grado de Educación Primaria de las asignaturas Matemáticas y su Didáctica II y III de los cursos lectivos 2018-2019 y 2019-2020 de una universidad pública española. El motivo de elegir estas dos materias radica en que es donde se encuentra la enseñanza-aprendizaje de las nociones aritméticas y geométricas. Los temas aritméticos en Educación Primaria son los que más trascendencia tienen (Montes et al., 2015), mientras que la geometría es el bloque matemático donde más errores se cometen (Nortes \& Nortes-Checa, 2017).

Tabla 1: Muestra con la que se ha realizado la propuesta. Fuente: Elaboración propia.

\begin{tabular}{cllc}
\hline Curso lectivo & Cuatrimestre & Asignatura & $N^{o}$ de alumnos \\
\hline $2018-2019$ & $1^{\text {er }}$ cuatrimestre & Matemáticas y su Didáctica III & 76 \\
$2018-2019$ & $2^{\text {o }}$ cuatrimestre & Matemáticas y su Didáctica II & 104 \\
$2019-2020$ & $1^{\text {er }}$ cuatrimestre & Matemáticas y su Didáctica III & 93 \\
$2019-2020$ & $1^{\text {er }}$ cuatrimestre & Matemáticas y su Didáctica III & 72 \\
\hline Total & & & 345 \\
\hline
\end{tabular}

\subsection{Percepciones de los estudiantes}

Consultados los docentes de la asignatura sobre las preocupaciones de su alumnado, se determina que las percepciones de los estudiantes de las asignaturas de matemáticas en el Grado de Educación Primaria son:

- Conocer si los cálculos que realizan están bien.

- Profundizar en diferentes procedimientos o métodos para la adquisición correcta de la noción, que en pocos años deberán trabajar a sus alumnos en el aula. 


\subsection{Desarrollo de la propuesta}

Dada la actual presencial de las TIC en los procesos de enseñanza aprendizaje, ya mencionados con anterioridad, se realizó una intervención en el aula con CalcMe y GeoGebra antes de pretender que los alumnos lo utilizasen de manera autónoma. La elección de estas dos aplicaciones viene motivada porque ambas son utilizadas en la actualidad en las aulas de Educación Primaria. Por todo ello, el conocimiento que los futuros docentes de Educación Primaria deben adquirir durante su periodo universitario debe considerar tanto a los contenidos didácticos como matemáticos.

En primero lugar, presentamos CalcMe (véase Figura 1) con el objetivo de ser utilizado para nociones aritméticas. Debido a su carácter intuitivo, se dedicaron algunos momentos en el aula habitual, sin necesidad de recurrir a un aula de informática. Los alumnos pudieron acceder desde sus ordenadores portátiles o teléfonos móviles. No fue necesario la realización de una formación adicional más allá de su presentación. En estos instantes, se focalizó el interés en la posibilidad de la manipulación de expresiones y el cálculo de las operaciones matemáticas que pudiesen aparecer en la asignatura para ser abordadas de manera autónoma, rápida e intuitiva.

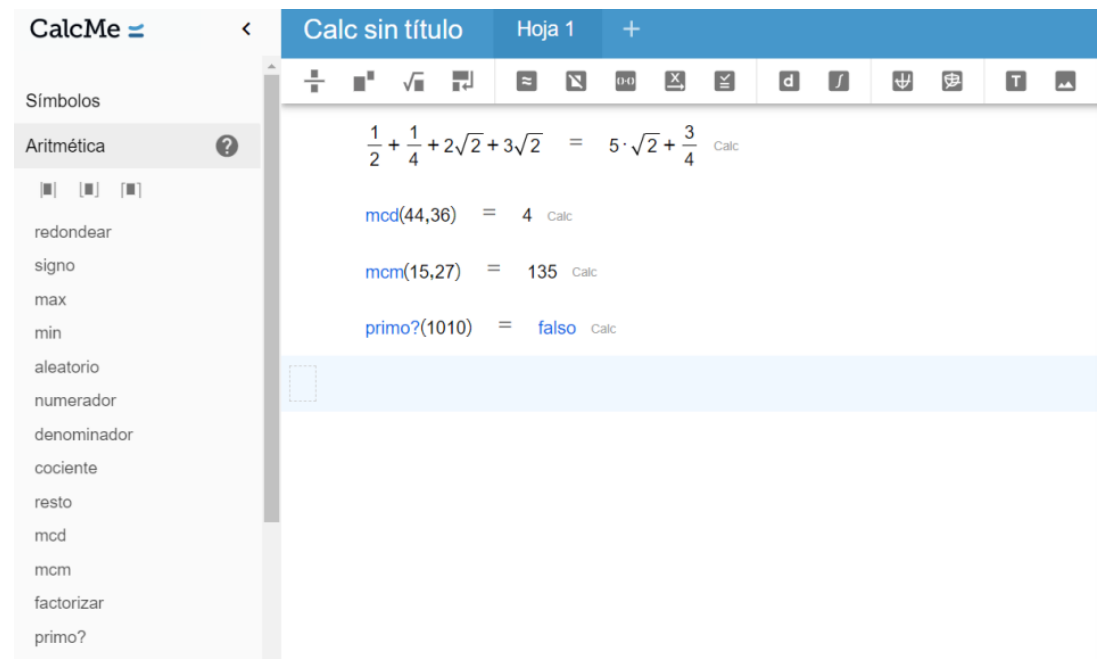

Figura 1: Ejemplo de visualización en CalcMe. Fuente: Elaboración propia.

En segundo lugar, GeoGebra (véase Figura 2) fue seleccionado por la posibilidad de construir figuras geométricas planas de manera dinámica e interactiva, además de nociones analíticas como las funciones relacionando diferentes sistemas de representación, algebraico y gráfico. En particular, para la construcción de figuras geométricas, funciones lineales y cuadráticas en la asignatura de Matemáticas y su Didáctica II, mientras que en la asignatura de Matemáticas y su Didáctica III se utilizó para la elaboración de transformaciones geométricas.

En este caso, sí fue necesario una formación específica llevada a cabo en un aula de informática. En esta se dio a conocer la potencialidad del software educativo, sus diferentes vistas, funcionamiento y dinamismo (véase Figura 3).

Este software no solo fue presentado como una propuesta útil para sus futuros alumnos, sino que también se expuso como ayuda en la adquisición de las nociones matemáticas que necesitan los futuros docentes antes de comenzar su labor docente.

Además de la formación presencial, se facilitó a los alumnos una secuencia de videos grabados por la docente de la asignatura acerca del uso de GeoGebra para las nociones involucradas en las asignaturas de Matemáticas y su Didáctica, de tal forma que cada uno pudiese continuar fuera del aula la tarea formativa.

A partir de este momento, los estudiantes universitarios crearon las tareas matemáticas que les permitiesen estar en una buena disposición ante su futura labor docente. Para ello, debían evaluar sus 


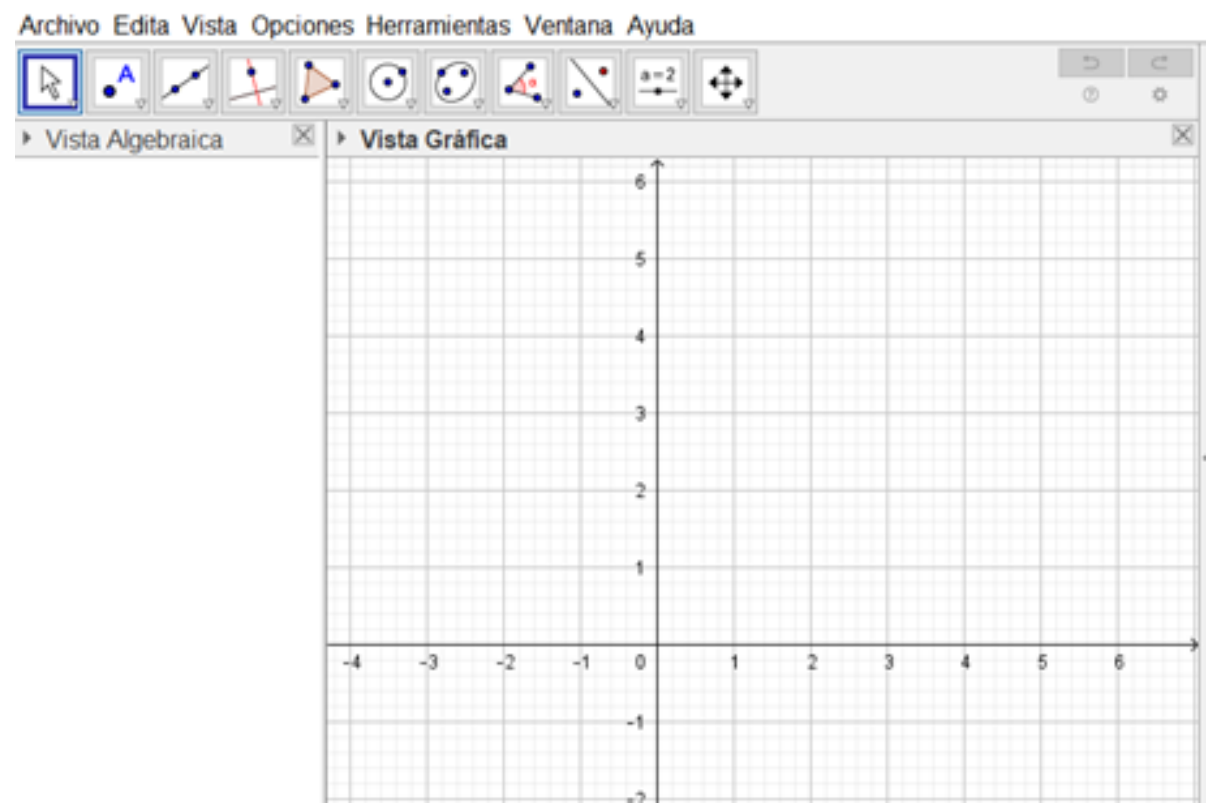

Figura 2: Pantalla de inicio de GeoGebra.

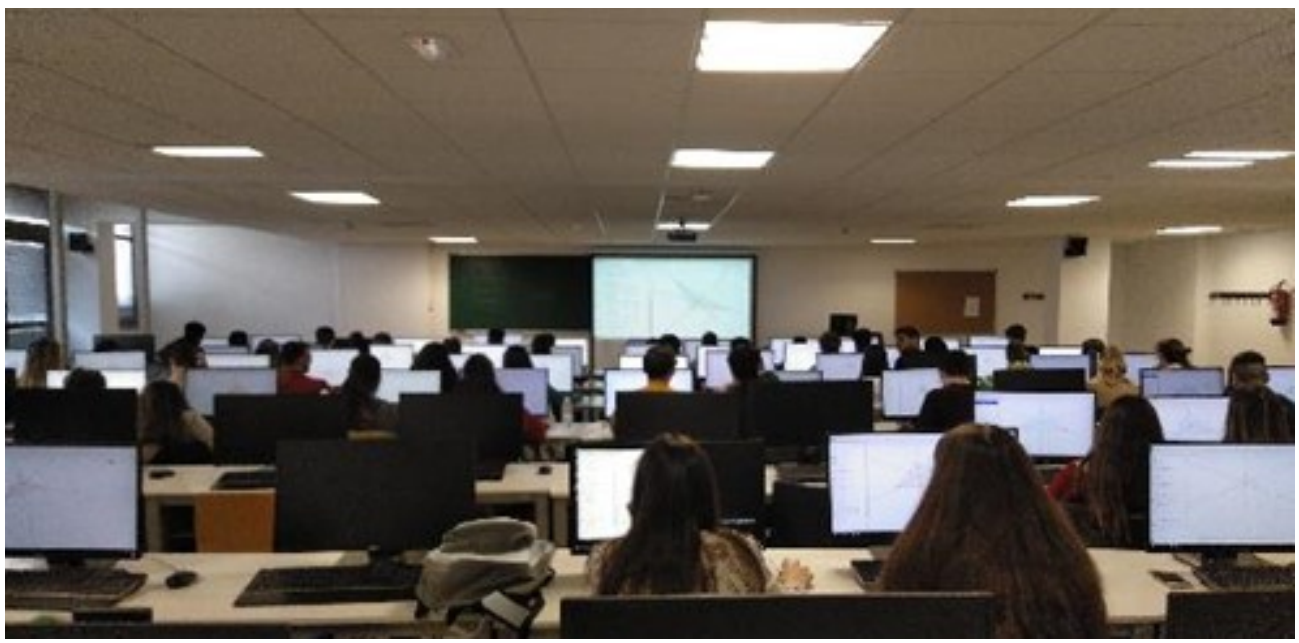

Figura 3: Aula de informática durante la formación presencial de GeoGebra.

conocimientos y ponerlos a prueba con el dinamismo que estas aplicaciones proporcionan.

La recolección de datos, para el caso de CalcMe, discurrió a partir de una observación participante completa (Spradley, 1980), ya que la mayor parte de ellas se han producido en el aula, y la entrega de una tarea que permitiese verificar el dominio del alumnado. Por otro lado, para GeoGebra se solicitó la entrega de distintos documentos en el Moodle de las asignaturas. En la asignatura de Matemáticas y su Didáctica II se les encomendó la entrega de dos tareas a través de Moodle: la construcción de la Recta de Euler y la cumplimentación de dos fichas de funciones cuadráticas, mientras que en Matemáticas y su Didáctica III se les solicitó la representación de transformaciones geométricas con único uso de punto medio, recta y compás de GeoGebra.

\section{Análisis de datos}

Las horas presenciales de cada una de las asignaturas de Matemáticas y su Didáctica en la universidad en las que se llevó a cabo el estudio eran 60. En cursos anteriores a la realización de esta propuesta, el escaso tiempo lectivo presencial dificultaba la resolución de todos los problemas y ejercicios pro-

Producción y evaluación de tareas matemáticas en las herramientas CalcMe y GeoGebra con los futuros docentes de Educación Primaria. Arnal-Palacián, M.

Derechos Reservados @ 2022 Revista digital Matemática, Educación e Internet (https://revistas.tec.ac.cr/index.php/matematica) 
puestos. Además, este alumnado demandaba, de manera directa al profesorado de las asignaturas involucradas, la necesidad de ampliar el número de ejercicios. El uso de las aplicaciones GeoGebra y CalcMe facilitó esta adaptación. Con el uso de ambas aplicaciones el alumnado fue capaz de producir tantas actividades como necesitase para desenvolverse de forma correcta con cada una de las nociones implicadas, incluso disminuir o aumentar el nivel de los ejercicios planteados.

A continuación, se muestran algunos ejemplos creados por los alumnos del Grado de Educación Primaria, ante la solicitud de las diferentes tareas.

A través de las operaciones aritméticas realizadas con CalcMe fue posible la corrección de diferentes ejercicios, tanto del resultado final como de los resultados intermedios, tal y como se muestra en la Figura 4. Esto propició un mayor aprovechamiento de las clases presenciales, dejando de lado el tiempo puramente algorítmico y que el alumno ya debiese conocer con anterioridad, dando lugar a una profundidad de las nociones matemáticas involucradas.

$$
\begin{aligned}
& \text { factorizar(48) }=2^{4} \cdot 3 \text { calc } \\
& \text { factorizar(27) }=3^{3} \text { calc } \\
& \sqrt{27}+\sqrt{48}=7 \cdot \sqrt{3} \text { calc }
\end{aligned}
$$

Figura 4: Ejemplo de operaciones aritméticas con CalcMe.

Con GeoGebra, en la tarea en la que debían construir la recta de Euler, Figura 5, los alumnos comprobaron la aleación de los siguientes puntos: el ortocentro, el circuncentro y el baricentro de un triángulo. Además, tuvieron la oportunidad de mover cada uno de los vértices, verificando que estos mismos puntos continuaban alineados en otra recta (véase Figura 6).

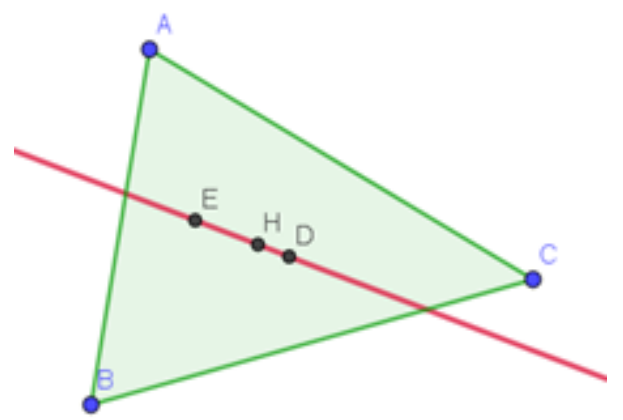

Figura 5: Ejemplo de Recta de Euler con GeoGebra.

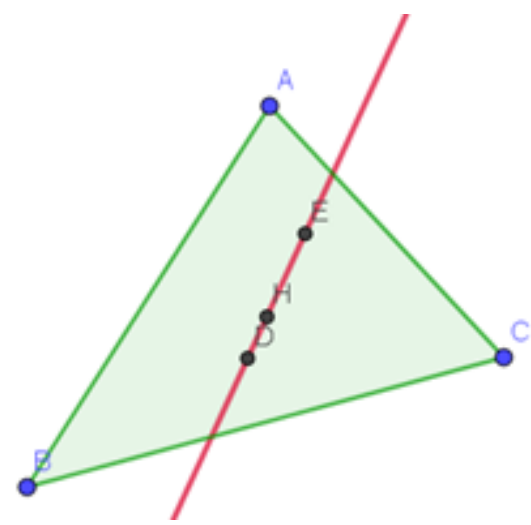

Figura 6: Ejemplo de Recta de Euler con GeoGebra después de mover los vértices. 
En el mismo bloque matemático, la geometría plana, fue posible la comprobación y la construcción de transformaciones geométricas, como, por ejemplo, la simetría axial (véase Figura 7), la simetría central o los giros.

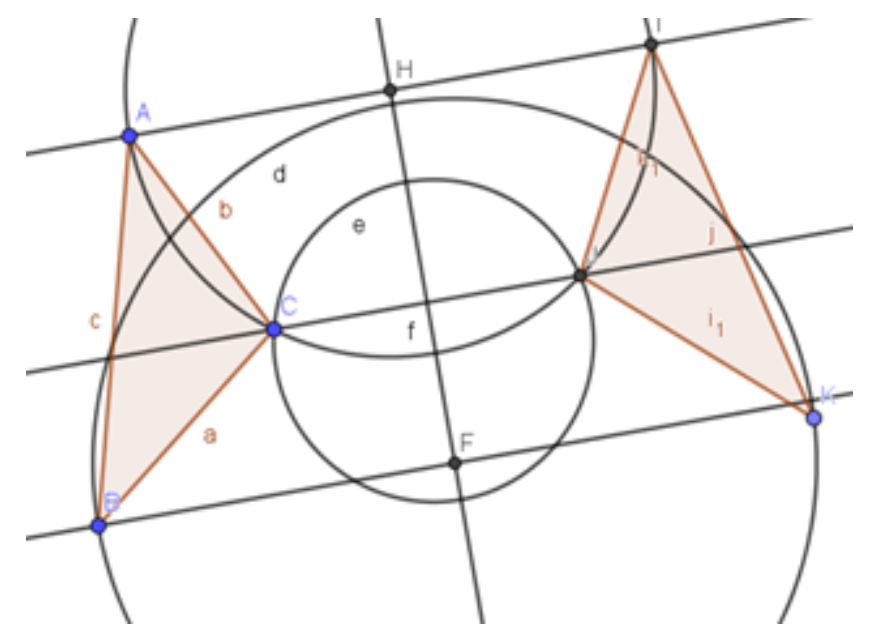

Figura 7: Ejemplo de transformación geométrica con GeoGebra.

Este dinamismo también fue comprobado a partir de la construcción de funciones cuadráticas, Figura 8. Observando como la modificación de cada uno de los coeficientes implicaba el cambio en la curvatura y en el vértice.

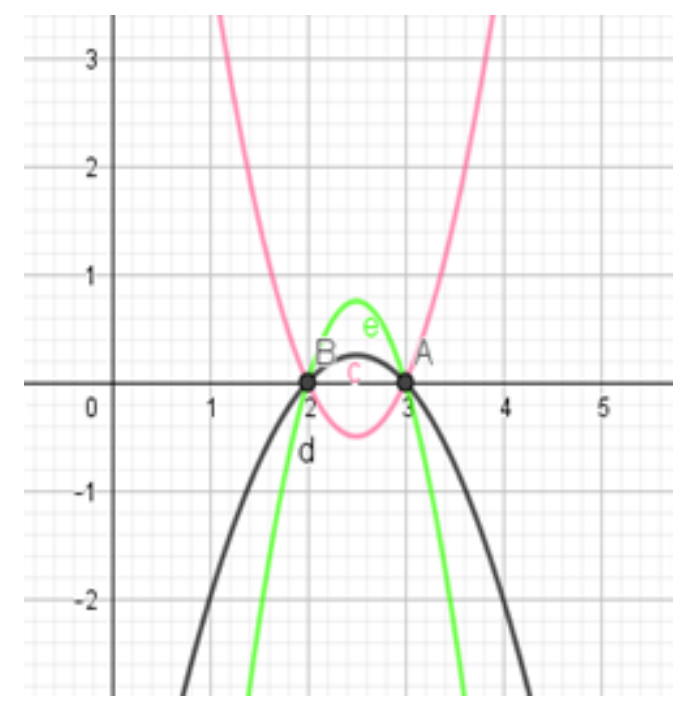

Figura 8: Ejemplo de funciones cuadráticas con GeoGebra.

Además, con el desarrollo de esta propuesta se han alcanzado los siguientes resultados:

- El alumnado de las asignaturas Matemáticas y su Didáctica II y III pudo autocorregir las tareas que involucraban a la aritmética, la geometría y el análisis matemático.

- El alumnado generó tantas tareas como necesitase para la adquisición del conocimiento necesario para un docente de Educación Primaria.

- El alumnado fue capaz de adaptar el nivel de las tareas a su propio aprendizaje.

- El alumnado desarrolló habilidades tecnológicas, necesarias para su futura labor docente.

- La docente de la asignatura pudo utilizar el tiempo de la resolución de algunas tareas matemáticas en otras de carácter didáctico, principal objetivo de la asignatura. 


\section{Conclusiones y perspectivas futuras}

A partir de esta experiencia fue posible la adquisición de las nociones matemáticas y didácticas previstas para las asignaturas Matemáticas y su Didáctica II y III del grado de Educación Primaria, logrando así el principal objetivo de esta propuesta de innovación docente.

Además, pudo verse mejorada la actitud, mediante la observación diaria en el aula, de la mayoría de los alumnos cuando tienen que enfrentarse a la incertidumbre de un resultado correcto en una tarea matemática.

Todo esto, junto con la creación de nuevas tareas en las que estaban involucradas la aritmética, la geometría y el análisis matemático, facilitó la adquisición de la comprensión de algunas nociones, al poder interpretar y experimentar a través de la simulación (Ngnoulaye \& Lepage, 2018). Además, pudieron ser adaptadas al nivel de cada uno de los alumnos en cada instante, pudiendo incrementar o disminuir la dificultad acorde a su conocimiento previo. Esto permitió un mejor aprendizaje individual (Saputra \& Fahrizal, 2019).

La introducción de dos aplicaciones informáticas, desconocidas para buena parte del alumnado con el que se realizó la experiencia, permitió desarrollar competencias digitales específicas en los futuros maestros de Educación Primaria, clave para una mejorara de la calidad educativa (Flórez et al., 2017).

Aunque se ha podido avanzar en la incursión de las nuevas tecnologías en la adquisición de conocimientos en diferentes asignaturas de Matemáticas y su Didáctica, hay que ser conscientes de que aún quedan tareas pendientes y, por tanto, la identificación de perspectivas futuras como, por ejemplo, trabajar de forma continuada con ambos softwares durante el transcurso de estas asignaturas y proseguir con la línea de esta experiencia en cursos lectivos posteriores.

\section{Reconocimientos}

Trabajo desarrollado en el grupo S60_20R-Investigación en Educación Matemática en el ámbito de la Comunidad Autónoma de Aragón para el periodo 2020-2022 y en el grupo HUM-324 del PAIDI de la Comunidad Autónoma de Andalucía.

\section{Bibliografía}

[1] Acosta, J. R. Uso del Software Wiris y su efecto en el Aprendizaje de límites e integrales en el curso de Matemática II en los estudiantes de Ingeniería Industrial del segundo ciclo de la Universidad César Vallejo. Trabajo Fin de Grado, Lima, Perú. 2017.

[2] Arnal, M., Baeza, M.A. \& Claros, J. Representación de funciones cuadráticas en $3^{\circ}$ ESO. Una propuesta que combina el uso de GeoGebra y el Pensamiento Matemático Avanzado. En L. J. Rodríguez-Muñiz, L. Muñiz-Rodríguez, A. Aguilar-González, P. Alonso, F. J. García y A. Bruno (Eds.), Investigación en Educación Matemática XXII (p. 609). Gijón: SEIEM. 2018.

[3] Arranz, J. M., Losada, R., Mora, J. A. \& Sada, M. Realidades de GeoGebra. Suma: Revista sobre Enseñanza y Aprendizaje de las Matemáticas, 67, 7-20. 2011.

[4] Benites, G., Herrera, A., Salas, C., Cuenca, J. Representación de las Funciones Trigonométricas en el Software Educativo como es el Programa Geogebra. Revista de Informática Educativa y Medios Audiovisuales, 6(12), 13-17. 2009. 
[5] Castillo, L., Gutiérrez, R. \& Prieto, J. L. Una perspectiva de análisis de las transformaciones geométricas en curvas de la función $\mathrm{f}(\mathrm{x})=$ eax utilizando el GeoGebra. Revista do Instituto GeoGebra de São Paulo, 2(2), 81-92. 2013.

[6] Fernández, J. \& Muñoz, J. Las TIC como herramienta educativa en matemáticas. Unión, 119-147. 2007

[7] Flórez, M., Aguilar, A.J., Hernández, Y.K., Salazar, J.P., Pinillos, J.A. \& Pérez, C.A. Sociedad del conocimiento, las TIC y su influencia en la educación. Revista Espacios, 38 (35), 39-51. 2017.

[8] García-Cid, Y., Marbán, M. \& Arnal-Palacián, M. A. Percepción de los estudiantes sobre el software GeoGebra en el estudio de la estadística en los grados de Educación. Anales de ASEPUMA, 28, 3. 2020.

[9] Gay, M., Tito, J. \& San Miguel, S. GeoGebra como facilitador del estudio de funciones de variable real. Congreso Iberoamericano de Ciencia, Tecnología, Innovación y Educación. 2014.

[10] Gutiérrez, R., \& Prieto, J. L. Deformación y reflexión de funciones con GeoGebra. El caso de las parábolas definidas por la expresión $\mathrm{g}(\mathrm{x})=$ ax2. Números. Revista de Didáctica de las Matemáticas, 88, 115-126. 2015.

[11] Hernández, C. Consideraciones para el uso del Geogebra en ecuaciones, inecuaciones, sistemas y funciones. Números. Revista de Didáctica de las Matemáticas, 82, 115-129. 2013.

[12] Huertas, A. \& Pantoja, A. Efectos de un programa educativo basado en el uso de las TIC sobre el rendimiento académico y la motivación del alumnado en la asignatura de tecnología de educación secundaria. Educación XX1, 19(2), 229-250. 2016.

[13] Iranzo, N. \& Fortuny, J. M. La influencia conjunta del uso de GeoGebra y lápiz y papel en la adquisición de competencias del alumnado. Enseñanza de las Ciencias, 27(3), 433-446. 2009.

[14] Losada, R. GeoGebra: La eficiencia de la intuición. La Gaceta de la RSME, 10(1), 223-239. 2008.

[15] Montes, M. Á., Contreras, L. C., Liñán García, M. D. M., Muñoz Catalán, M. C., Climent, N. \& Carrillo, J. Conocimiento de aritmética de futuros maestros. Debilidades y fortalezas. Revista de Educación, 367, 36-62. 2015.

[16] Ngnoulaye, J. \& Lepage, M. Influence des TIC sur l'apprentissage des étudiants à l'Université de Yaoundé 1 Influence of ICT on student learning at the Yaounde 1 university campus. Frantice.net, 14, pp.63-84. 2018.

[17] Nortes, R., \& Nortes-Checa, A. Competencia matemática, actitud y ansiedad hacia las Matemáticas en futuros maestros. Revista Electrónica Interuniversitaria de Formación del Profesorado, 20(3), 145-160. 2017

[18] Pascual, M.A. La nueva frontera educativa con nuevas tecnologías. Nuevas tecnologías, medios de comunicación y educación. Formación inicial y permanente del profesorado. Madrid: CCS. 1998.

[19] Ruiz, N., Bosch, M. \& Gascón, J. Modelización funcional con parámetros en un taller de matemáticas con Wiris. Sociedad, Escuela y Matemáticas. Aportaciones de la Teoría Antropológica de lo Didáctico (TAD). Universidad de Jaén: Jaén, España. 2007.

[20] Saputra, E. \& Fahrizal, E. The development of mathematics teaching materials through geogebra software to improve learning independence. Malikussaleh Journal of Mathematics Learning (MJML), 2(2), 39-44. 2019. 
[21] Spradley, J. P. Participant observation. New York: Holt, Rinehart and Winston. 1980.

[22] Suasnabas, L., Ortega, Ávila, W. F., Díaz, E. D. J. \& Rodríguez, V. M. Las Tics en los procesos de enseñanza y aprendizaje en la educación universitaria. Dominio de las Ciencias, 3(2), 721-749. 2017.

[23] Tamayo, E. D. Implicaciones didácticas de Geogebra sobre el aprendizaje significativo de los tipos de funciones en estudiantes de secundaria. Apertura, 5(2). 2013.

[24] Tirado, P. J., \& Roque, M.P. TIC y contextos educativos: frecuencia de uso y función por universitarios. Edutec. Revista Electrónica De Tecnología Educativa, (67), 31-47. 2019.

[25] UNESCO. Global Citizenship Education: Preparing learners for the Challenges of the 21st Century, Paris. 2014 